\title{
Health care practices of slum dweller adolescent girls in Bangladesh: The case of Sylhet city
}

\author{
Muhammed Muazzam Hussain \\ Professor, \\ Department of Social Work, \\ Shahjalal University of Science \& Technology, Sylhet \\ Dr. Mohammad Ali Oakkas \\ Associate Professor, \\ Department of Social Work, \\ Shahjalal University of Science \& Technology, Sylhet
}

\begin{abstract}
The aim of the paper is to know the health care practices of adolescent girls living in the slum areas of Sylhet city, Bangladesh, understand their health and hygiene issues including treatment, pregnancy, menstruation, vaccination, housing, water and sanitation related challenges they face in their daily lives. The study was based on primary sources of data; however the secondary data e.g. official documents, books, journals, policy papers etc. were used to conduct the study. The study followed qualitative research approach and the case study method to generate sufficient primary data. The study reveals that adolescent girls of slum areas face some menstruation related physical health problems e.g. abdominal pain, irregularity in menstruation cycle, excessive bleeding etc. Sometimes the guardian and doctor do not give much importance on it. The study findings show that adolescent girls of slum areas face multiple health care challenges including low level of personal hygiene, carry on treatment cost, lack of appropriate water, housing and sanitation facilities, etc. The study also highlights that family poverty, behavior of doctors, high cost of medicine, illiteracy of guardian etc. are responsible factors for low uptake of health care services. Therefore, the study suggests for coordinated efforts from the government, local government and voluntary agencies to upgrade their service delivery system.
\end{abstract}

Key words: Health care practice, adolescence girl, slum dwellers, Sylhet, Bangladesh

\section{INTRODUCTION}

Bangladesh has experienced a phenomenal growth in the rate of urbanization and total number of urban people. The people living in urban areas and cities constitute 35.86 percent of the total population (Statista, 2017). The growing process of urbanization also causes the increase of slum areas and people living there. Adolescence is regarded as a significant period of human growth and development which shape a person's future personality and potentialities. Adolescent are identified as those persons who belong to the age group of 10 and 19 years (WHO, 2014). The UNICEF Annual Results Report (2017) also recognized that millions of adolescent girls in low- and middle-income countries facing gender inequality along with poverty and other forms of discrimination and disadvantage and access to resources. There are 29.5 million adolescents in Bangladesh, including 14.4 million girls and 15.1 million boys, together representing nearly one-fifth of the country's total population of 144 million. However, the national health policy of Bangladesh (2011) did not give much focus on the issue of adolescent health and challenges, recently the government of Bangladesh has adopted a National Adolescent Health Strategy (2017-2030) to address the health care needs of 
adolescents by taking a broad and holistic approach for understanding health issues and care practices for them (MCH Services Unit, 2016). Despite some progress in current activities undertaken by the Ministry of Health and Family Welfare during the $7^{\text {th }}$ Five year plan (20162020 ) as it is recognized modernizing health care and expanding population and nutrition services are crucial for the development of the country, critiques argue that initiatives to address adolescent health care services provided by the Government of Bangladesh (GOB) and nongovernmental organizations (NGOs) remained fragmented and less evaluated and health services are often focused on clinical aspects rather than expanding opportunities for preventive measures (Ainul et al., 2017). It is well documented that slums population in developing countries like Bangladesh are facing multiple challenges including different types health problems and diseases, lack of basic services e.g. housing, water and sanitation, treatment, education and many other utility services i.e. gas and electric facilities, telephone, ambulance etc. (Rashid, Gani \& Sarker, 2013, Joshi, Fawcett, \& Mannan, 2011). The adolescent marriage and early childbearing is also another major health concern in Bangladesh where about one in four women age 20-24 had given the birth of their first child at the age of 18 (BBS, 2015). A recent study conducted by Chandra-Mouli \& Patel (2017) points out that in majority cases adolescent girls remained uninformed and unprepared for menarche. However, they get some information from mothers and other female family members, these does not ensure appropriate mental preparation to accept it as a common physical issue, rather feel shame which often lead to misconceptions and unhygienic practices during menstruation. Since, majority of the studies are conducted on the Dhaka city areas and there is a lack of studies in the North East region of the country i.e. the Sylhet division which is characterized by its diversity of environment e.g. famous for its hilly tea garden areas, wetland called haor basin and the plain land. In addition, it is distinct for growing urbanization, both internal and external migration causes a new formation of small and sporadic slum areas commonly known as koloni and Therefore, it is worthwhile to investigate the health care practices of adolescent girls living slum areas of Sylhet city, identify major issues and challenges they face while living in slum areas and propose some policy recommendations to improve their condition.

\section{OBJECTIVES OF THE STUDY}

The objectives of the study were twofold: First, to know the situation of health care practices of adolescent girls living in slum areas of Sylhet city and identify major challenges they face in their daily lives.

\section{STUDY DESIGN AND METHODOLOGY}

The study followed qualitative research approach and the case study method to generate sufficient primary data. This method was chosen because it was thought more appropriate to gain better understanding of the health condition, health belief, health care practices, and menstruation issues of the adolescent girls living there. The in-depth interviews were undertaken with semi-structured interview schedule to collect necessary information to address the research objectives. In addition, two focus group discussions were arranged with six to eight participants in each group to substantiate the interview data.

\section{The study area and population}

The Sylhet City Corporation was selected purposively as the area of study. It was chosen because, the growth of urbanization is much higher than any other city of Bangladesh except Dhaka and the urbanization process is very recent so that we can learn health care pattern of slum dweller adolescent girls. The other reason is that in Bangladesh majority NGOs and INGOs working for slum population and conducting studies focused on Dhaka cities, but there are a few researches have been conducted in the Sylhet city. According to the Bangladesh of Bureau of Statistics report (BBS, 2015), there are 670 slums in Sylhet city with a number of total 
population are 50129. However, the statistics do not figure out the total number of adolescent girls living in those slums.

\section{Sampling procedure}

Primarily three (03) slums were selected purposively selected for recruiting research participants from the Sylhet city corporation area on the basis of high density of slum dwellers. Twenty one (21) adolescences girls were selected from the three (03) slums under the ward no. 9, 13 and 15 and two FGDs were conducted with another two slums under the ward no. 3 and 5 of the Sylhet city corporation area. The following inclusion criteria were selected to recruit the research participants:

1. To be included in the study the participant should girl under the age of 12 to 19 years old and live in the slums, known as 'Koloni' for at least one year.

2. Literate and illiterate, married and unmarried girls of the above mentioned criteria could participate in the study.

\section{Data collection}

The interview protocol was designed in the line with established studies e.g. Rodehaver (2008). Open ended interview guideline were prepared and used to lead the interview session smoothly (Kerr and Smith, 2001). For focus group discussions, the ground rules were negotiated with the participants to make the discussions interactive. In many cases, questions were open ended with a number of sub questions, which were substituted for the following two main research questions:

1. What types of health care do they practice e.g. treatment, personal hygiene, water and sanitation etc.

2. What types of challenges do they face in health care practices?

On an average the duration of in depth interviews were 45 minutes to 1 hour and the FGDs were 2 hours to 2 hours and 30 minutes in length. The researcher along with two data collectors carried out the interviews and conducted the FGDs to understand health issues of adolescent girls, their health belief, health care issues including treatment pattern etc. The data collection process was ended when the interview or FG reached at the saturation point when no new data were found and the participants repeated the same information. The data collection period was six month from March, 2017 to September, 2017. A full transcription i.e. verbatim method was followed and the audio recorded interviews were first written in Bangla and then translated and typed into English.

\section{ETHICAL CONSIDERATIONS}

Since, there were no any institutional review board (IRB) to direct the research process in the Shahjalal University of Science \& Technology, the researcher followed the ethical guidelines offered by Miles and Huberman (1994). Participation in the study was entirely voluntary and relied on the ethical principle of informed consent. At the start of interview and FGD, the study purpose and nature was explained. They were asked to choice whether they can participate or not to participate and those who wanted to participate. A written consent was taken from those who can write and verbal consent was taken from those who did not know how to put signature.

\section{DATA ANALYSIS}

The in depth interviews and FGDs were audio recorded with the permission of the respondents. The verbatim recordings were transcribed and translated into English by the researcher and two research assistants. The data were read, re-read and taken for open coding and thematic analysis. From the original data codes were generated in terms of the various 
concepts and issues and then selective coding was conducted to systematically analyze the data and identify the most prominent themes. The 'iterative' analysis process was followed and a particular attention was given to choosing and using the quotations which is most relevant to the specific issue (Chen, 2013).

\section{Socio-demographic information}

\section{THE FINDINGS}

Majority of the participants of in-depth interview belong to the age group of 12- 19 years for in-depth interview and 16- 19 years for FGDs. Adolescents below 12 years were excluded from interview and FGD for their tender age. In terms of religion, it is shown that majority of them belong to the religion of Islam. The data on family type shows that majority of them belong to the single family and family size remains 4 to 6 members. Regarding the information education, it is shown that majority of FGD participants completed primary education and Interview participants completed secondary level education. The information about housing reveals that majority of them live in Kaccha houses, made of bamboo and corrugated iron sheet or brick and corrugated iron sheet. The family income varies from Bangladeshi Taka 7000.00 to $20,000.00$

\section{Health care practices: Treatment}

The study findings show that majority of the respondents go to the public hospital for receiving health care services where they can consult with a doctor with a very little cost. But they face some challenges to avail the services. The following narratives echo the issue:

"Once I was admitted into the Osmani Medical College Hospital for gastric ulcer. But there are a very limited number of seats for the patients. I had to share my bed with other woman patient. After two days I got my own bed."(Interview 05, Age: 13 years) "For only long term acute diseases I go to the Osmani Medical College Hospital. But I don't like to go there, because I need to stand long hours to see the doctor. The doctors pay less attention to us, they write the prescription without listening carefully." (Interview 11, Age: 15 years)

"The problem is that patients need to wait for long hours to meet with the doctor. Medicines are not free; only cheap medicine e.g. paracetamol tablet is provided free. The cost of medicines is much expensive and it is impossible for us to buy all medicines." (FGD-2, Married AG)

The study findings also demonstrate that there are some respondents who receive services from the private hospital or clinic, because it is near to their slum, but they cannot continue their full course of treatment due to high cost involved with it. The following extracts echo the issue:

"Once my menstruation was continuing for 18 days, that time I have consulted with a doctor in the Ragib Rabeya Medical College. The doctor prescribed lots of medicines but I couldn't complete the course, because the medicines were much expensive."(Interview 02, Age: 17 years)

"If the disease is simple and hospital outdoor services are required then I can afford, but when it is necessary to admit in the hospital, then we cannot bear the expenses. During my delivery I was admitted there. Though it was normal delivery, I had to pay much money."(Interview 12, Age: 19 years) 
The study demonstrates that a significant number of parents of adolescent girls usually seek medical help from local traditional healers and quacks for their adolescent girls due to lower costs involved with that treatment. The following narratives raise the issue:

"Once I was affected by stomach pain and my parents took medicine from a local quack. But taking the medicines I felt vomiting and fever. Then they took me to the nearest public hospital and they told that previous treatment was wrong." (Age: 17 years)

"My mother relies on taking tabiz from local imam of the mosque for removing my headache and abdominal pain. My father goes to medical vendor and takes some painkiller for this. I try to follow both the instructions to get cure from the diseases." (Age: 15 years)

The study findings also reveal that sometimes adolescent girls get allopathic treatment for diarrhea and malaria fever, but prefer kabiraji treatment (herbal treatment) for jaundice and skin diseases. The narratives clarify the issue:

"Once I suffered from itching problem and diseases and went to kabiraj for treatment. He suggested using the leaf of tree to remove my itching problem and now I feel well."(Interview 11, Age: 15 years)

"I took 'Jarfuk' treatment from Kabiraj for jaundice. The kabiraj gave me some herbal medicines which I had to put on my head after cutting hairs. I had to keep it for more than one week. After few days of the treatment I got cured." (FGD 01)

"Basically I take allopathic medicines from the nearest pharmacy for treatment. Sometimes I seek Kabirazi treatment. Once my menstruation was continuing for 15 days, then I met a Kabiraj and took 'Tabij' from him." (Interview 01, Age: 15 years).

The data demonstrates that some guardians of adolescent girls rely on homeopathic treatment for menstruation related disease but sometimes they do not get the expected positive outcome. The following narratives point out the issue:

"My menstrual period is not regular. Besides I also feel abdominal pain during the menstruation period. I had taken homeopathic medicines for more than two years but didn't get any fruitful result."(FGD 01)

"I am facing vaginal discharge problem after the birth of my son. He is now three years old. At first I didn't think it's a disease. When I felt continuing headache and weakness, I went to homeopathic doctor and took medicines, but still do not get cure." (FGD 02, Married Girl)

\section{Health care during pregnancy}

The study findings show that some respondents and their family members are not aware to receive health care during pregnancy. The following narratives clarify the situation:

"During my pregnancy I had consulted with the doctor only one time. My husband said it's not so essential to meet with doctor regularly, pregnancy is not a disease. My mother-in-law also didn't support to consult with the doctor." (Interview 06, Age: 18 years)

"During my pregnancy I didn't take nutritious food due to poverty. So my health condition was not good and I was very weak. Because of my weakness I needed to get a surgery and birth of a under weight baby."(FGD 02) 
The study also reveals that some married adolescent girls depend on traditional birth attendants 'Dai' and local 'Kabiraj' for treatment. One FG respondent shared her experience in the following way:

"When I was pregnant I have consulted with the doctor only one time. At the time of the birth of the baby, my family brought a 'Dai' to help me in delivery. Because of her inexpert delivery, my baby son was born abnormal." (FGD, 02)

"Due to poverty the people in slums prefer to call a traditional birth attendant. My mother in law also called a 'dai' at time of my delivery. But after delivery of baby I am facing many health challenges e.g. vaginal white discharge, urinary problems etc." $(F G D, 02)$

\section{Menstruation issue}

The study reveals that adolescent girls of slum areas face some menstruation related physical health problems e.g. abdominal pain, irregularity in menstruation cycle, excessive bleeding etc. The following extracts echo the issue:

"I face abdominal pain along with acidity before and after starting the menstruation. I have consulted with the doctor about the abdominal pain but the doctor said it will recover after marriage." (Interview 02, Age: 17 years)

"Sometimes my menstruation starts lately, after 8 or 10 days late. When menstruation starts lately it takes more time such as 7 or 8 days and get much bleeding. Normally it takes 3 or 4 days to complete the menstruation period in a month. I didn't consult with the doctor." (FGD 01)

The study also point out that a large number of adolescent girls had no idea about the menstruation period before starting it. Because the family members e.g. their mother, elder sister did not share anything before starting the menstruation. The following excerpts echo the issue:

"I just knew that menstruation was a disease of women. Before starting menstruation, I felt serious abdominal pain and became afraid when I saw bleeding. Then my mother and friends talked with me details about menstruation." (Interview 03, Age: 15 years)

"During menstruation I feel abdominal pain, vomiting and sometimes feel very cold or hot in my body. When I came to know from my friends that menstruation is a shameful disease of woman, I didn't talk with anybody." (Interview, Age: 15 years)

"As I did not go to school, I had no idea about the menstruation. So when my first menstruation started I became tensed and nervous. I couldn't share this with anybody." (Interview 03, Age: 15 years).

The study found that some of the adolescent girls are facing problems of excessive vaginal discharge. One respondent shared her experience in the following way:

"After birth of my first child, I felt excessive vaginal discharge problem. When it was started it continued for 6 or 7 days and I felt very weak and serious headache." (Interview 12, Age: 19 years)

"I am suffering from excessive virginal discharge for more than three years. At first, I didn't take it as a serious matter. Now I am afraid, I feel headache, breast pain and weakness for that problem. I didn't share the problem with anyone due to shy." (FGD 02) 
"Before starting my menstrual period in every month I used to feel vaginal discharge problem. It started two or three days before starting menstruation. I felt weakness and muscular pain in my body." (Interview 01, Age: 15 years).

\section{Personal hygiene challenges}

The study points out that majority of the adolescent girls of slum areas do not practice the rules of personal hygiene strictly. The following narratives raise the issue:

"I wash my hands before or after taking meal. I also wash my hands after going out from the toilet. I forgot to wash my hands when I took dry food and I don't think it's important to wash hands always while taking dry food." (Interview 12, Age: 19 years)

"I follow the general health and cleanliness rules at the time of taking meal. I wash my hands with soap after going out from the toilet. But sometimes I forgot to carry soap along with me while going to toilet which is far away from my house." (FGD -01)

"There are very dust and garbage in our slum. I know using sandal is always hygienic. But I do not feel comfort to use sandal always. I like to walk without sandal and I think it is the reason of my diarrheal diseases." (Interview 06, Age: 13 years)

The study shows that majority of adolescent girls use 'Rags' commonly known as Nekra instead of sanitary napkin which are washed quickly (with a small plastic tub of water called 'bodna' inside the latrine. There is no private place to change and clean the rags and often no safe water and soap to wash them properly. The following narratives clarify the issue:

"I use rag as an alternative to sanitary pad during menstruation. I can't use sanitary pad because these are very costly. After using it many times I have thrown it to the dustbin. I clean that piece of cloth with only normal water as using soap or detergent is most expensive." (Interview 01, Age: 15 years)

"I can't use sanitary pads, because of its high cost. I can't clean the rags at day time because both male and female members of the slum bath in the same tube-well. I have to wait for night to wash the changing cloth or used rags."(Interview 11, Age: 15 years)

Even in their homes, a culture of shame forces women to find well-hidden places to dry the rags. These places are often damp, dark and unhealthy. One participant from FGD group shared her opinion in the following way:

"I use rags at the time of menstruation. I use one rag 7 or 8 times by washing it every month. I do not use hot water to clean the rags. I kept the using cloths inside the room for dry. I never kept the rag in the sun, because it's a matter of shy. My mother said to keep these matter secret from male persons because these are secret issues of girls". (FGD 01)

\section{Vaccination}

The study findings show that majority of the adolescent girls are not conscious about vaccination e.g. tetanus or others vaccines and only few respondents completed the all doses of TT vaccine. The following narratives clarify the issue:

"I have taken two doses of tetanus vaccine. I didn't complete the other doses because I have no idea about the importance of TT vaccine. I thought I can take others doses anytime when I want."(Interview 08, Age: 15 years) 
"I took only two doses of TT vaccine from the health workers who came to visit our slum. After providing two doses they stopped to visit our slum and I forgot to complete it. (Interview 03, Age: 15 years)

"I have taken only two doses of Tetanus vaccine when the health workers of NGO came to our school. At the time of third doses, they didn't come to our school and I forgot to take it." (Interview 10, Age: 14 years)

The study findings also show that schools going adolescent girls have some knowledge about different vaccination, but they cannot receive due to poverty. The following extracts clarify the issue:

"I have received three doses of Tetanus and Ham Vaccines by the health workers who come to visit our school. I wanted to take Hepatitis-B vaccine but could not afford it due to high cost." (Interview 07, Age: 13 years)

"I have learnt the utility of different vaccination from my school teachers. I have completed all doses of TT vaccine from the Sylhet Osmani Medical College hospital at free of cost. I wanted to get vaccine of Uterus Cancer, but it was highly costly which I could not avail." (FGD 01)

The study also found that there are some married adolescent girls who knew the importance of vaccination and wanted to receive it but cannot get the opportunity, because their mother in laws did not approve it. The following narratives raise the issue:

"I am pregnant but I didn't receive any vaccine till now. Health workers suggest me to take vaccine. I have tried many times to make my mother-in law understand about the importance of vaccination. But she said, it is not essential, she had 5 children and never received any vaccine." (FGD 02)

"There are some misconceptions prevail in our slum that by vaccination the health condition of pregnant women become vulnerable and require surgery for birth of a baby. Therefore, some guardians e.g. mother in law oppose it and show reason that they did not take vaccines at their time and they were well." (Married AG, 18 years)

\section{Housing, water and sanitation}

The study reveals that majority of the adolescent girls of slum live in inappropriate housing settings and overcrowded condition. The following narratives clarify the issue:

"Most of the houses of our slums are kaccha i.e. made of mud, straw and bamboo which produce much fiber particles and do not protect us from the cold or heat waves which increases asthma related health problems."

"There are seven members in our family and we live in a large room. Our house is kaccha and made of bamboo. We do not have any privacy there."

The study findings also show that water and sanitation condition of the slum is very poor. Most of the adolescent girls of the slums use tube-well for the purpose of drinking, bathing, toileting, cooking and household needs etc. But one tube-well in one slum is not sufficient to fulfill the water needs of the residents and as there are no separate tube-well facilities for male and female, the adolescent girls cannot maintain privacy there. The following excerpts echo the issue:

"There is one tube well in our slum. When it is damaged, I use pond water without boiling. So, it could be a cause of jaundice for me." (Interview 02, Age: 17 years) 
There is a tube well in the slum. I took my bath from it. There is a curtain of polythins surrounding the tube well. But there is no privacy for us. (Interview 01, Age: 15 years)

"There are two toilets in our slum which has no cover. Most of the residents do not use much water after defecation as the only source of water i.e. tube well is far away from the toilet." (Interview 08, Age: 15 years)

\section{Treatment experiences}

\section{DISCUSSION}

The study findings show that majority of the respondents go to the public hospital for receiving health care services where they can consult with a doctor with a very little cost. But they face some challenges to avail the services. For example, there are a very limited number of seats in the public hospital for the patients. The adolescent girls need to share bed with other woman patient which they do not feel comfortable. Sometimes they need to wait one or two days a get a bed. The other challenges include long waiting hours both for outdoor and indoor services and lack of attention of doctors. It is evident from the study that sometimes they are not given sufficient time to listen their health problems and the cost of medicine is also high which in many times they cannot afford. The study findings also demonstrate that there are some respondents who receive services from the private hospital or clinic, because it is near to their slum, but they cannot continue their full course of treatment due to high cost involved with it.

The study findings also reveal that sometimes adolescent girls get allopathic treatment for diarrhea and malaria fever, but prefer 'kabiraji' or herbal treatment for jaundice and skin diseases. The kabiraj or traditional healers provide with 'jarfuk' and some herbal medicines for treatment of jaundice. For menstruation problem, they sometimes prefer to go traditional healers e.g. local religious person who provide 'tabiz' and 'pani pora' etc. The findings also demonstrates that some guardians of adolescent girls rely on homeopathic treatment for menstruation related disease but sometimes they do not get the expected positive outcome. This study mirrors the findings of other study which found that more than 75 per cent slum dwellers receive allopathic treatment 12 per cent receive kabiraji (i.e. herbal or traditional or religious healing) and 9 per cent use homeopathic treatment (Nahid et al, 2015).

\section{Health care during pregnancy}

The study findings show that some respondents and their family members are not aware to receive health care during pregnancy. Their health seeking behavior e.g. visiting doctor, health check up and uptake of health care services etc. is governed by husband and mother in law etc. who do not give much importance about this due to illiteracy, unconsciousness or negligence. The family poverty is also responsible for low uptake of health care services and malnutrition or anemia of married pregnant adolescent girls of slums in Sylhet city of Bangladesh. The study also reveals that some married adolescent girls depend on traditional birth attendants 'Dai' and local 'Kabiraj' for treatment of pregnancy related complexities. For example, in one case it is found that depending on traditional birth attendant also result in head injury of baby and ultimately became associated with child disability.

\section{Menstruation related challenges}

The study reveals that adolescent girls of slum areas face some menstruation related physical health problems e.g. abdominal pain, irregularity in menstruation cycle, excessive bleeding etc. Sometimes the guardian and doctor do not give much importance on it and suggest that it will recover after marriage. The study also point out that a large number of adolescent girls had no idea about the menstruation period before starting it. Because the family members e.g. their mother, elder sister did not share anything before starting the menstruation. Therefore, some misconception grows among the adolescents that it is a shameful disease of woman and one 
should not share about this with anybody. More specifically the dropped out or illiterate adolescent girls do not have right information about this and they become tensed and nervous when their first menstruation start. The study also found that some of the adolescent girls are facing some additional challenges e.g. problems of excessive vaginal discharge which is associated with some other symptoms including headache, breast pain and physical weakness etc. But the crucial thing is that they do not share the problem with anyone or seek any treatment due to shy which poses the risk of their reproductive health.

\section{Personal hygiene}

The study findings also demonstrates that majority of the adolescent girls of slum areas do not practice the rules of personal hygiene strictly. They wash their hands before or after taking meal and also wash their hands after defecation. By contrast, they forget to wash hands in taking dry food and do not think the importance of washing hands while taking dry food. It is also evident from the study that some of them forget to carry soap while going to common toilet or washroom which is far away from their houses and they do not always clean their hand after defecation by soap. This might have an impact of causing diarrhea and dysentery disease among the slum dweller adolescent girls. It is also found that a significant number of adolescent girls in the slum do not use sandal and remain bare footed in day time.

The study findings also reveal that the vast majority slum girls in Sylhet city use rags instead of sanitary pad or napkin due to high cost involved with it. These rags are made of torn part of old saris. They face additional difficulties to make it clean as there are is no private place to change and wash it because they need to use a common toilet where soap or cleaning materials are not available. By contrast, it is found that adolescent feel shy to bring nekra and soap with them to go to the toilet. Even in their own homes, they struggle hard to find a well-hidden place to dry the rags. These places are often damp, dark and unhealthy which poses a big challenge to maintain hygienic practice and safe reproductive health.

\section{Vaccination}

The study findings show that majority of the adolescent girls are not conscious about vaccination e.g. tetanus or others vaccines and do not give much importance on completion of full doses. Some of them thought they can take other doses at any convenient time. The study findings also show that schools going adolescent girls have some knowledge about different vaccination but they cannot receive those are not provided at free of cost due to poverty. For example, they have taken Tetanus and Ham Vaccines provided free of cost by public health workers but cannot afford to take Hepatitis-B vaccine or vaccine of Uterus Cancer which are not provided free of cost. The study also found that there are some married adolescent girls who knew the importance of vaccination and wanted to receive it but cannot get the opportunity, because their mother in laws did not approve it.

\section{Housing, water and sanitation challenges}

The present study shows that water and sanitation facility is very poor in the slum areas of Sylhet city and the adolescent girls face some crucial health care challenges e.g. lack of pure drinking water, latrine facilities, overcrowded housing etc. It is evident from the study that some adolescent girls use tape water which was supplied from Sylhet City Corporation. The condition of water supply is not clean; however, there is found small slab in the bathroom which stands in an open place and widely used for the purpose of bathing and daily household activities. These bathrooms are open place which are even visible from the road and both male and female members of the neighborhood use it. So the adolescent girls cannot maintain privacy. Another important challenge they face include unhygienic condition of bathroom and high risk involved in using it as the slab is always slippery and covered with moss. This place is 
also used for the purpose of cooking and washing things. These entire situations portray a potential threat to their physical health and general wellbeing.

\section{CONCLUSION}

The study findings demonstrate that they constitute one of the vulnerable segments of population in our country due to poverty, malnutrition, inappropriate housing condition, lack of pure and safe water, sanitation etc. The study findings also demonstrates that majority of the adolescent girls of slum areas do not practice properly the rules of personal hygiene and not much conscious about vaccination. and face difficulties due to poor housing, water and sanitation problem. However, majority of them seek modern allopathic treatment method, some of them seek alternatives e.g. herbal or kabirazi, homeopathic etc. The study identified some reasons including the high cost involved with modern treatment, long waiting hours to receive health care from public hospitals and lack of responsiveness and attention of doctors etc. which act as barriers to get access and utilization of public health care services. Consequently, majority of slum dweller adolescent girls prefer to go to local pharmacies for primary treatment. By contrast, a significant number of them rely on traditional healing method as an alternative to allopathic treatment. The study findings also demonstrates that majority of the adolescent girls of slum areas do not practice properly the rules of personal hygiene and not much conscious about vaccination and other health care aspects including adolescent pregnancy. Therefore, it is suggested that health care and hygiene practices of the slum dweller adolescent girls can be improved through coordinated efforts by the different government departments, city corporation authority and NGOs working in the areas.

\section{References}

Ainul, S., Bajracharya, A., Reichenbach, L., \& Gilles, K. (2017). Adolescents in Bangladesh: A situation analysis of programmatic approaches to sexual and reproductive health education and services. Washington, DC \& Dhaka, Bangladesh: Population Council.

BBS. (2015). Census of Slum Areas and Floating Population 2014. Dhaka: Bangladesh Bureau of Statistics, Statistics and Informatics Division, Ministry of Planning, Government of the People's Republic of Bangladesh.

Chandra-Mouli, V., \& Patel, S. V. (2017). Mapping the knowledge and understanding of menarche, menstrual hygiene and menstrual health among adolescent girls in low-and middle-income countries. Reproductive health, 14(1), 30.

Joshi, D., Fawcett, B., \& Mannan, F. (2011). Health, hygiene and appropriate sanitation: experiences and perceptions of the urban poor. Environment and Urbanization, 23(1), 91-111.

MCH Services Unit. (2016). National Adolescent Health Strategy (2017-2030) Ministry of Health and Family Welfare, Government of the People's Republic of Bangladesh, Dhaka. Retrieved from: http://coastbd.net/wpcontent/uploads/2017/07/National-Strategy-for-Adolescent-Health-2017-2030-Final-Full-Book-21 Date: 04-032019.

Miles, M.B., and Huberman, A. M., (1994). An Expanded Sourcebook: Qualitative Data Analysis. Thousand Oaks, CA: Sage.

Rashid, S. F., Gani, S., \& Sarker, M. (2013). Urban poverty, climate change and health risks for slum dwellers in Bangladesh. In Climate Change Adaptation Actions in Bangladesh (pp. 51-70). Springer, Tokyo.

Statista. (2017). Retrieved from https://www.statista.com/statistics/455782/urbanization-in-bangladesh/ Date: 03-03-2019

The 7th Five Year Plan, (2016-2020). Accelerating Growth, Empowering Citizens. General Economics Division, Planning Commission, Government of the People's Republic of Bangladesh, Dhaka.

The national health policy of Bangladesh (2011) The 7th Five Year Plan, (2016-2020). Accelerating Growth, Empowering Citizens. General Economics Division, Planning Commission, Government of the People's Republic of Bangladesh, Dhaka.

The National Health Policy, (2011). Ministry of Health and Family Welfare, Government of the People's Republic of Bangladesh, Dhaka. 
UNICEF Annual Results Report (2017) Retrieved from: https://www.unicef.org/publications/index_102865.html Date: 04-03-2019.

UNICEF. (2011). The state of the world's children 2011-adolescence: an age of opportunity: United Nations Children's Fund (UNICEF).

WHO, 2014. Health for the World Adolescents, A second chance in the second decade, Retrieved from https://apps.who.int/adolescent/second-decade/files/1612 MNCAH_HWA Executive Summary.pdf Date: 20.11.2019. 